\title{
Wavelet-based option pricing: An empirical study
}

\author{
Xiaoquan $\mathrm{Liu}^{*} \quad \mathrm{Yi} \mathrm{Cao}^{\dagger} \quad$ Chenghu $\mathrm{Ma}^{\ddagger} \quad$ Liya Shen ${ }^{\S}$
}

July, 2018

\begin{abstract}
In this paper, we scrutinize the empirical performance of a wavelet-based option pricing model which leverages the powerful computational capability of wavelets in approximating risk-neutral moment-generating functions. We focus on the forecasting and hedging performance of the model in comparison with that of popular alternative models, including the stochastic volatility model with jumps, the practitioner Black-Scholes model and the neural network based model. Using daily index options written on the German DAX 30 index from January 2009 to December 2012, our results suggest that the wavelet-based model compares favorably with all other models except the neural network based one, especially for longterm options. Hence our novel wavelet-based option pricing model provides an excellent nonparametric alternative for valuing option prices.
\end{abstract}

Keywords: Pricing; Option Valuation; Artificial Neural Networks; Stochastic Volatility; Jump Risk

JEL codes: G12, G13

\footnotetext{
* Nottingham University Business School, University of Nottingham Ningbo China, 199 Taikang East Road, Ningbo, 315100, China. Email: Xiaoquan.Liu@Nottingham.edu.cn. Financial support from the INQUIRE UK Grant 2007-06 is gratefully acknowledged.

${ }^{\dagger}$ Management Science and Business Economics Group, University of Edinburgh Business School, 29 Buccleuch Place, Edinburgh EH8 9JS, UK. Email: jason.caoyi@gmail.com.

${ }^{\ddagger}$ Fudan University Management School, Shanghai, China. Email: machenghu@fudan.edu.cn.

${ }^{\S}$ Corresponding author. Essex Business School, University of Essex, Colchester CO4 3SQ, UK. Email: lshenb@essex.ac.uk. Phone: +44 1206874679.
} 


\section{Introduction}

Since the seminal work of Black and Scholes (1973), huge progress has taken place in the theoretical and empirical option valuation literature that has greatly advanced our understanding of the options market as a place for trading information and gauging investor expectation. A large number of parametric and nonparametric methods have been developed to relax one or more restrictions of the original Black-Scholes model.

One avenue for extending the Black-Scholes model is to develop nonparametric models that are better at capturing the volatility smile and the literature has seen innovative methods in this direction. Wavelets are well-known for their remarkable ability in numerical approximation and the wavelet-based option pricing model developed in Ma (2011) leverages this. It approximates the implied risk-neutral moment-generating functions (MGF) thus offering a novel approach in the nonparametric option pricing literature. Unlike many other nonparametric option pricing models that require a large collection of data, the wavelet-based pricing model is computationally efficient and requires only a reasonable amount of different strikes. Using numerical experiments, Haven et al. (2009) demonstrate that this model is able to evaluate and forecast option prices with great precision.

In this paper, we contribute to the literature by taking this further to empirically compare the forecasting and hedging performance of the wavelet-based model with three other wellestablished models, namely the parametric stochastic volatility model with jumps (SVJ), the practitioner Black and Scholes model (PBS), and the hybrid neural network based model (NN), which is a combination of the neural network method and the Black-Scholes model. ${ }^{1}$ We focus on the key research questions of whether the excellent performance of the wavelet-based model in simulation still remains in the crucial test using market data, and how its empirical performance compares with that of widely-accepted models in the literature.

To the best of our knowledge, this is the first study that subjects the wavelet-based option pricing model to market data. We use daily index options written on the DAX-30 index, a

\footnotetext{
${ }^{1}$ We thank an anonymous referee for the suggestion of adding the practitioner Black-Scholes model and the neural network based model for comparison.
} 
major financial index in Europe, from January 2, 2009, to December 28, 2012. Our main empirical findings can be summarized as follows. In the out-of-sample forecasting exercises ranging from one- to ten-day ahead, the wavelet-based model outperforms the SVJ for calls and exhibits similar performance for puts for all option maturities (short-, medium- and long-term) across all forecasting horizons. It also outperforms the PBS model in forecasting long-term options. This is the case regardless of whether the market is in a turbulent state with evident jumps in the underlying stock index. The NN model is always the best-performing model. In the hedging exercise, the performance of the wavelet-based model is second only to that of the NN and exhibits substantially smaller hedging error than the SVJ and PBS model. ${ }^{2}$ Our strong empirical evidence substantiates the wavelet-based option pricing model as a credible alternative in the option valuation literature.

The rest of the paper is organized as follows. In Section 2, we review the relevant literature that motivates our study. Section 3 introduces the wavelet-based option pricing model, the SVJ model, the PBS model, and the neural network based model and how we conduct the hedging exercise. In Section 4, we describe data and analyze empirical results. Finally, Section 5 concludes.

\section{Literature review}

In this section, we review two strands of the literature to which our paper makes a contribution: the literature of option valuation and that of the wavelet method and its applications.

In the parametric option pricing literature, researchers have identified a number of priced factors essential in capturing the volatility smile, which has become a stylized fact since the market crash of 1987. For example, volatility is shown to relate negatively to the underlying asset returns and that delta-hedged portfolios of options and the underlying stocks produce statistically significant negative returns (see, for instance, Bakshi and Kapadia (2003), Coval

\footnotetext{
${ }^{2}$ We have conducted the same empirical analysis using options written on the FTSE 100 index in the UK and the Hang Seng index in Hong Kong over the same sample period. We obtain qualitatively similar results that we do not report to conserve space. These are available upon request from the authors.
} 
and Shumway (2001), Heston (1993), and Wong and Lo (2009)). Moreover, the random and unexpected jumps are also found to command significant risk premium in the options market (Bates, 1996, 2000; Cai and Kou, 2011; Pan, 2002). Another priced factor worth noting is the demand pressure in the market, which affects option prices in incomplete markets (Gârleanu et al., 2009).

Bakshi et al. (1997) propose a closed-form parametric option pricing model that simultaneously admits the stochastic volatility risk, the jump risk, and the stochastic interest rate risk. One or more risks can be singled out by setting the parameters of the remaining risk factors to zero so that the importance of each risk factor can be closely investigated. Based on the pricing, forecasting, and hedging performance of nested models, they show that the stochastic volatility and jumps are of first-order importance when it comes to accommodating the volatility smile observed in the market.

Another prominent option pricing model is the practitioner Black-Scholes model (PBS). In PBS, a nonlinear deterministic volatility function (DVF) is employed to estimate volatility values and account for deviations from the assumptions of the original Black-Scholes model (Derman and Kani, 1994a,b; Rubinstein, 1994; Dumas et al., 1998). A theoretical justification of the PBS approach as a reduced-form approximation of an unknown structural model is provided by Berkowitz (2002). As discussed in Andreou et al. (2014), the PBS model is widely used among practitioners as it is effective in mitigating the volatility smile anomaly and easy to implement (Christoffersen and Jacobs, 2004; Christoffersen et al., 2009; Berkowitz, 2010).

Parallel to the intensive interest in the parametric option pricing literature, a large number of nonparametric models have also been developed. Although the nonparametric models lack the economic interpretation that the parameters contain in the parametric family of models, they are often more flexible as they impose no prior assumption on the underlying asset process. These include the flexible distribution method (Rubinstein, 1994), the cubic spline method (Shimko, 1994), which is further developed by Bliss and Panigirtzoglou (2002), the kernel estimation method (Aït-Sahalia and Lo, 1998; Aït-Sahalia and Duarte, 2003; Birke and Pliz, 2009), the neural network method (Hutchinson et al., 1994; Garcia and Gençay, 2000; Andreou et al., 
2008), and the $\epsilon$-arbitrage replicating portfolio method (Bandi and Bertsimas, 2014).

The neural network method is a popular method in option pricing. It is first introduced by Hutchinson et al. (1994) to estimate the unknown pricing formula for derivative securities and further employed for pricing derivatives in Lajbcygier et al. (1996), Garcia and Gençay (2000), and Yao and Li (2000). They all show that when sufficiently trained, neural network method can adapt to changing market conditions and achieve remarkable accuracy. Andreou et al. (2008) combine the neural network method and the parametric option pricing models and show that the combined hybrid neural network model works even better than the pure neural network method. The neural network methods are highly data-intensive and require large amount of historical prices to obtain a reasonably well-trained network.

A more recent addition to this growing literature is Ma (2011). This book develops a nonparametric option pricing model that focuses on approximating the implied risk-neutral MGF of the underlying asset returns using wavelets. The risk-neutral MGF has a number of advantages compared with the implied risk-neutral PDF although there is a one-to-one relationship between them. For example, the MGF is more tractable when jumps are present in the underlying price process; the MGF obtained from options is a continuous function; the MGF can be used to obtain all the statistical moments of the underlying asset distributions and the preference parameter of the utility function; and out-of-sample options with different maturity dates can be directly estimated using the risk-neutral MGF. ${ }^{3}$

Ma (2011) represents another effort in applying the wavelet method, already a popular tool in science and engineering, in the area of economics and finance. ${ }^{4}$ As pointed out in Haven et al. (2009) and Haven et al. (2012), there are mainly three types of application of wavelet methods in finance and economics. First of all, wavelets are used for multi-scaling analysis. For example, Ramsey and Lampart (1998a,b) use the wavelet method to analyze the relationship between economic variables at different scales and suggest that the relationship changes over different time horizons. Gençay et al. (2001a,b, 2003, 2005) employ the wavelet multi-scaling approach

\footnotetext{
${ }^{3}$ See Haven et al. (2009) for detailed properties of the risk-neutral MGF.

${ }^{4}$ For excellent reference for applications of the wavelet method in finance and economics, see Gençay et al. (2002). See also Percival and Walden (2000) for applications of the wavelet method in the time series analysis.
} 
to examine intra-day seasonalities, foreign exchange volatilities, and systematic risk. Weron (2009) implements the wavelet method to de-seasonalize electricity prices. More examples include Zapart (2002), Connor and Rossiter (2005), Kim and In (2005), Mitra (2006), In and Kim (2006), Fernandez (2006), Lien and Shrestha (2007), Gallegati and Gallegati (2007), and Nikkinen et al. (2011).

Secondly, wavelets are used to de-noise raw data. Capobianco $(1999,2001)$ show that wavelets as a pre-processing de-noising tool are useful for improving volatility analysis. The superior de-noising ability of wavelets is also recognized in Haven et al. (2012) which apply the wavelet method to de-noise option prices before estimating the implied risk-neutral PDF from the option prices. Their findings show that the wavelet de-noising process significantly improves the density estimation quality and the forecasting abilities of the estimated densities. Sun and Meinl (2012) substantiate the superior performance of the wavelet-based local linear scaling approximation algorithm in denoising high-frequency financial data. Asgharian (2011) de-noise frequency variations in the first principal component of a business cycle with wavelets. Other research in this stream includes, among others, Averkamp and Houdré (2003) and Lada and Wilson (2006).

Finally, wavelets are utilized to estimate unknown parameters of a model. For example, Jensen $(1999,2000)$ and Ko and Vannucci (2006) adopt wavelets for calibrating parameters of long memory processes. Genon-Catalot and Laredo (1992) apply wavelets in estimating a diffusion function non-parametrically. Matache et al. (2005) adopt wavelets to price Americanstyle options driven by Lévy processes. Manchaldore et al. (2010) implement the wavelet method to obtain intraday volume. Ortiz-Gracia and Oosterlee (2016) use Shannon wavelet to price European options. Additional references include Bayraktar et al. (2004), Hong and Kao (2004), Dong and He (2007), Esteban-Bravo and Vidal-Sanz (2007), and Haven et al. (2009). 


\section{Model specifications}

In this section, we outline the wavelet-based option pricing model of Ma (2011), the SVJ of Bakshi et al. (1997), the PBS model of Dumas et al. (1998) and Andreou et al. (2014), and the hybrid neural network method of Andreou et al. (2008). We also discuss how we perform the hedging test.

The wavelet-based option pricing model

The wavelet-based model by Ma (2011) is the latest theoretical contribution to the option pricing literature. Its motivation and relation to the option-implied risk-neutral MGF and the option-implied risk-neutral PDF are discussed in Haven et al. (2009) and Ma (2011).

Under fairly general assumptions including i.i.d. distribution for asset returns, the waveletbased option pricing model can be expressed as follows:

$$
C_{t}\left(S_{t}, X, T\right)=X e^{-r(T-t)} \mathcal{L}^{-1}\left(\frac{\Theta^{T-t}(s)}{s(s+1)}\right)\left(\ln \frac{X}{S_{t}}\right)
$$

where $\mathcal{L}^{-1}$ denotes the bilateral inverse Laplace transform ${ }^{5}, C_{t}$ is the time $t$ price for a European call option written on asset whose price is $S_{t}$ with strike price $X$ and a future maturity date $T$. Interest rate $r$ and the dividend yield are assumed to be constant.

The core of this pricing model is $\frac{\Theta^{T-t}(s)}{s(s+1)}$, where $s$ is a complex value whose real part $\operatorname{Re}(s)<-1$ for calls and $\operatorname{Re}(s)>0$ for puts. The MGF $\Theta^{T-t}(s)$ of the logarithmic returns $\ln \frac{S_{T}}{S_{t}}$ captures the underlying asset dynamics and investor expectation embedded in option prices, and needs to be approximated with wavelets.

To approximate the implied MGF with the wavelet method, a particular wavelet needs to be chosen from a large family of wavelet functions. The wavelet literature seems to agree that there is no best wavelet for a particular application. Therefore, we follow Mallat (1999) and choose a wavelet that can achieve a reasonable level of accuracy with minimum number of wavelet terms. The Franklin hat function performs well on this criterion. In addition, it has

\footnotetext{
${ }^{5}$ See Appendix for the definition and properties of the bilateral inverse Laplace transform.
} 
the properties of being symmetric, smooth, and piecewise continuous, and it closely emulates the probability density function of asset returns.

The risk-neutral MGF $\Theta(s)$ of the return per unit of time is therefore estimated using the Franklin hat function $h(t)$, which is defined as follows:

$$
h(t)=\left\{\begin{array}{cc}
(1-|t|) & \text { if }-1 \leq t<1 \\
0 & \text { otherwise }
\end{array} .\right.
$$

The Laplace transform of $h(t)$ is denoted as $m_{h}(s)$ :

$$
m_{h}(s)=\left(\frac{e^{s / 2}-e^{-s / 2}}{s}\right)^{2} .
$$

A set of generalized functions can be generated from the Franklin hat function $h(t)$ :

$$
h_{l, k}(t)=2^{\frac{l}{2}} h\left(2^{l} t-k\right), l, k=0, \pm 1, \pm 2, \ldots
$$

The scaling parameter $l$ determines the degree of dilation or contraction and the shifting parameter $k$ controls the horizontal location of the function. Perform Laplace transform on $h_{l, k}(t)$, we obtain $m_{l, k}(s)$ as follows:

$$
m_{l, k}(s)=2^{-\frac{l}{2}} e^{-\frac{k s}{2^{l}}} m_{h}\left(\frac{s}{2^{l}}\right), l, k=0, \pm 1, \pm 2, \ldots
$$

The risk-neutral MGF of the return per unit of time $\Theta(s)$ can be expanded using the Laplace transform of the set of generalized Franklin function as follows:

$$
\Theta(s)=\sum_{l=-\infty}^{\infty} \sum_{k=-\infty}^{\infty} a_{l k} m_{l, k}(s),
$$

where $a_{l, k}$ is a set of unknown coefficients and needs to be estimated by minimizing the sum of squared error between market option prices and theoretical prices. We follow the procedure in Haven et al. (2009) and estimate the unknown coefficients as follows. 
1. Truncate the coefficients $a_{l k}$ by setting $a_{l k}=0$ for all $|l|>L$ and $|k|>K$, where $L$ and $K$ are positive integers. ${ }^{6}$ Let $\theta_{L, K} \equiv\left\{a_{l k}\right\}_{l=L,|k| \leq K}$.

2. Given a collection of market data for options at time $t,\left\{S_{t}, X_{i}, C_{t, i}, T, r\right\}$, where $i=$ $1,2, \ldots, N$, we estimate the unknown coefficients $\theta_{L, K}$ by minimizing the sum of squared errors between market option prices $C_{t, i}$ and theoretical prices $\hat{C}_{t, i}$ :

$$
\min _{\theta_{L, K}} \Sigma_{i}\left(C_{t, i}-\hat{C}_{t, i}\left(\theta_{L, K}, S_{t}, X_{i}, T, t, r\right)\right)^{2}
$$

3. Increase $L$ by 1 at a time and repeat the above steps until $\sum_{i}\left(C_{t, i}-\hat{C}_{t, i}\right)^{2}<\varepsilon$ for an arbitrary $\varepsilon>0$

The above optimization process yields an estimate of the risk-neutral MGF, which is expressed as a series of the Laplace transform of the set of the generalized Franklin functions:

$$
\hat{\Theta}(s)=\sum_{|l|=L} \sum_{|k| \leq K} \hat{a}_{l k} m_{l k}(s) .
$$

In the empirical analysis, the scaling parameter $L$ and the shift parameter $K$ are chosen by the optimisation programme so that a satisfactory estimation result can be obtained.

The stochastic volatility model with jumps (SVJ)

The volatility and jump risks have long been considered priced factors in the options market and should be included in option pricing models (Coval and Shumway, 2001; Bates, 1996; Huang and Wu, 2004; Pan, 2002; Santa-Clara and Yan, 2010). Bakshi et al. (1997) formulate a parametric model that incorporates a mean-reverting stochastic volatility component that correlates with the underlying stock and a jump process that follows the Poisson distribution.

Assuming constant interest rate, the closed-form formula for European call options is as follows,

$$
C_{t}=S_{t} \Pi_{1}\left(t, T, S_{t}, V_{t}\right)-X \exp (-r(T-t)) \Pi_{2}\left(t, T, S_{t}, V_{t}\right),
$$

\footnotetext{
${ }^{6}$ According to Haven et al. (2009), $K$ is chosen to be the smallest integer greater than $0.7 \times 2^{l}+1$ as $\log$ returns typically lie between $[-0.7,0.7]$. The value of $K$ can be easily adapted to specific situations.
} 
where the risk-neutral probabilities $\Pi_{1}$ and $\Pi_{2}$

$$
\Pi_{j}\left(t, T, S_{t}, V_{t}\right)=\frac{1}{2}+\frac{1}{\pi} \int_{0}^{\infty} \operatorname{Re}\left[\frac{\exp \left(-i \phi \ln (X) f_{j}\left(t, T, S_{t}, V_{t} ; \phi\right)\right.}{i \phi}\right] d \phi, j=1,2
$$

are obtained by inverting the characteristic functions $f_{1}$ and $f_{2}$. Note that the expression in the square brackets is a complex number and $R e[\cdot]$ takes the real part of it.

There are a number of parameters in the SVJ model. The jump process is described with the mean jump size $\mu_{J}$, the standard deviation of jump size $\sigma_{J}$, and the jump frequency $\lambda$. The mean-reverting stochastic volatility process $V_{t}$ are parameterized by the speed of adjustment $\kappa_{v}$, the long-term mean of the volatility $\theta_{v} / \kappa_{v}$, and the variation coefficient of the diffusion volatility $\sigma_{v}$. The volatility process and the underlying asset dynamics are correlated with coefficient $\rho$. For index options and most equity options, $\rho$ is negative corresponding to the negative skewness found in the risk-neutral distributions. In addition, the underlying assets of many different types of options exhibit volatility mean reversion which is also captured in this SVJ model (Bali and Demirtas, 2008; Wong and Lo, 2009). These unknown parameters are calibrated by minimizing the sum of squared differences between market option prices $C_{t}$ and theoretical ones $\hat{C}_{t}$.

The practitioner Black-Scholes model (PBS)

Although the Black-Scholes (BS) option valuation framework is still widely used by practitioners due to its reasonable performance and straight-forward structure, the implied volatility of the BS model, however, has been the focus of intense research. The regression-based deterministic volatility function (DVF) Dumas et al. (1998) and Andreou et al. (2014), is one of the popular approaches for determining option specific volatilities to accommodate the volatility smile or smirk observed in market data.

In this study, we select two best-performing structures of DVF in Dumas et al. (1998) and Andreou et al. (2014) as follows:

DVF 1: $\sigma=\max \left(0.01, a_{0}+a_{1}(\ln X)+a_{2}(\ln X)^{2}\right)$ 
DVF 2: $\sigma=\max \left(0.01, a_{0}+a_{1}(\ln X)+a_{2}(\ln X)^{2}+a_{3} T+a_{4}(\ln X) T+a_{5} T^{2}\right)$.

DVF 1 expresses volatility as a simplified function of $\log$ strike price $\ln X$ whereas DVF 2 specifies volatility as a function of $\log$ strike price $\ln X$ and option time to maturity $T$. These two specifications are termed PBS1 and PBS2, respectively.

We follow the literature and employ the nonlinear least square (NLS) regression to estimate the model parameters. In the NLS, the sum of squared errors between the model price and the market price is minimized by the Levenberg-Marquardt method, which interpolates between the Gauss-Newton algorithm and the gradient descent method. We implement this using the minpack $\mathrm{R}$ package.

The hybrid neural network based model (NN)

We follow Andreou et al. (2008) to construct an artificial neural network (NN) based model for option pricing. As one of the most popular data-driven models, NN operates as a nonlinear regression tool as follows:

$$
Y=G(\tilde{x})+\epsilon_{N N}
$$

that maps the unknown relation, $G(\cdot)$, between the input variables, $\tilde{x}=\left[x_{1}, x_{2}, \ldots, x_{N}\right]^{T}$, and the target function $Y$. The traditional NN usually implements the Multi-layer Perceptron (MLP) with a single hidden layer for mapping the inputs with the target function, and the existing literature reaches the consensus that a single hidden layer is sufficient to make MLP a universal approximator for most problems (Bengio, 2009).

An MLP with a single hidden layer is a function $f: R^{D} \rightarrow R^{L}$, where $D$ is the size of the input vector $\tilde{x}$, and $L$ is the size of the output vector $Y$, such that the total approximation function can be represented as follows:

$$
Y=G\left[b^{(2)}+W^{(2)}\left(S\left(b^{(1)}+W^{(1)} x\right)\right)\right]
$$

where $b^{(1)}$ and $b^{(2)}$ are bias vectors, $W^{(1)}$ and $W^{(2)}$ are weight matrices, and $G(\cdot)$ and $S(\cdot)$ are 
activation functions. The function $\Phi(x)=S\left(b^{(1)}+W^{(1)} x\right)$ constitutes the hidden layer, where $W^{(1)}$ is the weight matrix connecting the input vector to the hidden layer. The output vector $Y$ is obtained as $G\left(b^{(2)}+W^{(2)}(\Phi(x))\right)$.

In this paper, we follow the specification in Andreou et al. (2008) for the hybrid neural network model. This NN model has a traditional three-layer structure: an input layer, a single hidden layer, and an output layer. The numbers of neurons in the input and the output layers are equal to the dimensions of the input and the output vectors $\tilde{x}$ and $Y$, respectively. The number of neurons in the hidden layer is between five to ten and determined by the cross validation. For the NN model, the input vector $\tilde{x}$ is constructed as as follows:

$$
\tilde{x}=\left[\left(S e^{-\delta T}\right) / X, T, r, \sigma\right]^{T},
$$

where $\delta$ is the dividend yield, and $\sigma$ is the volatility. The $\sigma$ is obtained through calibrating the implied structural parameters by focusing on the Brownian volatility to drive the residual error to zero (Andreou et al., 2008). The output target vector in $\mathrm{NN}$ is represented as follows:

$$
C_{t, i} / X-C_{t, i}^{B S} / X
$$

where the estimated BS call prices are obtained when volatility is proxied by historical volatilities. When training the NN model, we employ the Levenberg-Marquardt method incorporated into the backpropagation algorithm (Hagan and Menhaj, 1994) to obtain efficient and accurate testing results. We implement the NN model by the neuralnet $\mathrm{R}$ package.

For this NN model, we use six months of data for training and another two months for cross validation. It is then used for forecasting option prices over the next one, two, five and ten days. The training and cross validation window then rolls forward one day following a rolling window scheme. This method is in line with Fig. 2 in Andreou et al. (2008) with Ts equal to one day. The rolling window scheme is able to capture the dynamics of the distribution but suffers from computational inefficiency (Ren et al., 2016). 


\section{$\underline{\text { Hedging exercise }}$}

In addition to out-of-sample forecasting, we also compare the hedging performance of the models following Hutchinson et al. (1994), Bakshi et al. (2000) and Garcia and Gencay (2000). Suppose we sell one call option and undertake $\Delta$ shares of stock and bonds to discretely hedge this call during its life. Let $V_{t}$ denote the value of the portfolio consists of the option, the stock and the bond as follows:

$$
V_{t}=V_{S, t}+V_{B, t}+C_{t},
$$

where $V_{S, t}, V_{B, t}$, and $C_{t}$ are, respectively, the value of the stock, the bond, and the call option held in the portfolio on date $t$. The initial value of the portfolio can be specified as follows:

$$
\begin{aligned}
V_{S, t} & =S_{t} \Delta_{t} \\
\Delta_{t} & =\frac{\partial \hat{C}_{t, T}}{\partial S_{t}} \\
C_{t} & =-\hat{C}_{t, \tau} \\
V_{B, t} & =-\left(V_{S, t}+C_{t}\right),
\end{aligned}
$$

where $\hat{C}_{t, T}$ is the call price estimated by a particular model on day $t$ with maturity $T$. Since we assume the stock purchase is entirely self-financed by the riskless borrowing and the sale of the call option, the initial value of the portfolio on date $t$ is zero: $V_{t}=V_{S, t}+V_{B, t}+C_{t}=0$. Before option expiry, we re-balance the stock and bond positions at regular interval $\Delta \tau$ to satisfy the following relations:

$$
\begin{aligned}
V_{S, t+\Delta \tau} & =S_{t+\Delta \tau} \Delta_{t+\Delta \tau} \\
\Delta_{t+\Delta \tau} & =\frac{\partial \hat{C}_{t+\Delta \tau, T-\Delta \tau}}{\partial S_{t+\Delta \tau}} \\
C_{t+\Delta \tau} & =-\hat{C}_{t+\Delta \tau, T-\Delta \tau} \\
V_{B, t+\Delta \tau} & =e^{r \Delta \tau} V_{B, t},
\end{aligned}
$$

where $\Delta \tau$ is chosen to be 7 or 30 days following Bakshi et al. (2000). The hedging error is, as of the revision day, the present value of the replicating portfolio value and can be expressed as 
follows:

$$
\epsilon=V_{B, t+\Delta \tau}+V_{S, t+\Delta \tau}+C_{t+\Delta \tau} .
$$

When we implement the hedging exercise for NN, we train and cross validate the model with data in the first eight months and use the remaining data for hedging performance evaluation without re-training the model.

\section{Data and empirical analysis}

In this paper, we use options written on the German DAX-30 index for our empirical investigation. Daily call and put option data from January 2, 2009, to December 28, 2012 are obtained from Ivolatility.com. Option prices are calculated as the average of end-of-day bid and ask prices, which avoids the bid-ask bounce. We take the Euro LIBOR rate as the interest rate. These data and dividend yields are obtained from the Datastream. The interest rates with five different maturities from one month to 12 months are matched with the options data based on maturities. The underlying DAX index is dividend-adjusted. We apply conventional exclusion rules to clean the raw options data, including:

- At- or in-the-money (ITM) options are removed;

- Options with prices below unity are removed;

- Options with less than 14 days to maturity or more than 365 days to maturity are excluded;

- Options with less than 10 contracts traded on a day are excluded;

- Options with less than 9 different strike prices with the same maturity are removed as we need sufficient strikes for parameter estimation.

These exclusion rules leave us with 65,867 calls and 95,895 puts over 1004 business days.

Descriptive statistics for the data are summarized in Table 1. Following Bates (1996), we divide the options into three categories. Short-term options have 90 days or less before expiry; medium-term options are between 90 to 180 days to maturity; and long-term options 
are between 180 and 364 days to expiry. In this way we avoid weighting longer-term options more heavily than shorter-term options (Huang and $\mathrm{Wu}, 2004$ ), which contain slightly different market information (Bakshi et al., 2000). Following Bakshi et al. (2000), we also group options according to their moneyness, defined as X/S. As time to maturity increases, we see proportionately more OTM options. For calls, the proportional of OTM options goes up from 53\% from short-term to $73 \%$ of long-term ones; whereas for puts, the corresponding proportions are $69 \%$ and $79 \%$ for short- and long-term options, respectively.

Our out-of-sample forecasting exercise is carried out on a rolling basis from 1 September, 2009, until the end of the sample period. On each day, all options with the same maturity date are used to estimate model parameters, which are then used as inputs to forecast option prices for a range of forecasting horizons from one day to ten days. The out-of-sample forecasting performance is reported in Table 2 for call options and in Table 3 for put options. We adopt three metrics to evaluate forecasting errors: the root mean squared error (RMSE), the mean absolute error (MAE), and the mean percentage error (MPE).

The first thing we notice is that the neural network based model outperforms all other models in offering the smallest forecasting errors in most cases. For example, in the one-day ahead forecasts for slightly OTM short-term calls in Table 2, the RMSE, MAE, and MPE for the neural network model is $3.95,2.37$, and 0.02 , respectively, much smaller than the corresponding errors produced by the other models. When we move to longer forecasting horizons with two-, five- and ten-day ahead forecasts, the neural network model continues to fare better than the other models across different maturities. For example, the error for medium-term five-day ahead put option forecasts are 1.86, 1.27 and 0.03 , respectively, for the RMSE, MAE, and MPE in Table 3, the lowest among the alternative models when options are slightly OTM.

However, as discussed in the previous section, neural network models are data-intensive and require a large amount of historical data to obtain a reasonably well-trained network. In this paper, we use six months of option data for training and another two months for cross validation before any forecasts are produced. This is different from the other methods whereby we only need one day's data to forecast prices for the following day and over longer forecasting 
horizons. Hence the neural network based model is not exactly comparable on an equal footing with other models in this regard.

Apart from the neural network based model, the wavelet-based model consistently outperforms the SVJ model for all three loss functions and across option maturities in Table 2 for call options. Similar performance for put options is reported in Table 3. For example, the MAE for ten-day ahead forecasts for the deepest OTM options are 9.05, 16.63, and 22.11, respectively, for short- (Panel A), medium- (Panel B) and long-term (Panel C) calls in Table 2. The corresponding values are $9.57,18.25$, and 24.27 , respectively, for the SVJ model.

When compared with the two PBS specifications, the wavelet-based model tends to fare better for long-term options but worse for short- and medium-term options across the forecasting horizons. For PBS2, the better-performing PBS model, its MPE remains 0.07 across the four forecasting horizons when moneyness is between 0.94 and 0.97 for long-term puts in Table 3 . This is higher than or equal to the MPE for the wavelet-based model at 0.03, 0.04, 0.05, and 0.07, respectively, over the same forecasting horizons. In addition, for these two tables in Panel $D$ we group OTM options by moneyness only to provide an overall picture. We observe the same patterns that the neural network based model performs best, and that wavelet-based model outperforms the SVJ model.

Our results are related to Andreou et al. (2014) which show that the PBS2 specification outperforms the SVJ in the out-of-sample forecasting test. However, their results are based on the use of all options simultaneously and the performance for options with different maturities is not examined. In this paper, we conduct our empirical analysis by looking at options per maturity in the spirit of Bakshi et al. (2000), which argue that the market information captured by short- and long-term options is somewhat different. Interestingly, our forecasting results differ a little per maturity. We find that the SVJ model indeed performs worse than the PBS2 for short- and medium-term options but it tends to outperform the PBS2 for long-term call options across the forecasting horizons. We also show that the wavelet-based options tend to perform better than the PBS model for long-term options in the forecasting exercise.

Furthermore, following Andreou et al. (2014) we conduct a subsample analysis to scrutinize 
the models when the market is in a volatile condition defined as when the underlying DAX index jumps by more than $1 \%$ in either direction. The results, summarized in Table 4 , are broadly consistent with our baseline results. We note that the neural network based model is still the best-performing model, that the wavelet-based model still outperforms the SVJ models across option maturities in Panels $A$ to $C$ and forecasting horizons from one to ten days, and that it outperforms the PBS model only for long-term options when the forecasting horizon is less than ten days. Panel D aggregates options only by moneyness and shows the same pattern.

In addition to the out-of-sample forecasting performance, we further examine the hedging performance between the option pricing models. The hedging errors, the average after each re-balancing, are reported in Table 5. Unsurprisingly, the neural network based method again offers the smallest hedging errors among all the models considered in this paper. Apart from this model, the wavelet-based model consistently outperforms other models across different option maturities and re-balancing frequency. For example, for short-term DAX options, the hedging error of the wavelet-based model is only -9.58 for seven-day re-balancing for the deepest OTM options, compared with -15.88 for the SVJ, and -14.18 for the two PBS specifications. The hedging error is only -8.07 for this group of options using the neural network based model.

It is worth mentioning that although in our paper the neural network based model comprehensively outperforms other models, in the literature however it is argued that this model does not perform very well for deep OTM or long-dated options. Bennell and Sutcliffe (2004) and Gradojevic et al. (2009) address this by categorizing options based on moneyness and time to maturity. They train nine modular neural networks for each group of options with improved performance at the cost of computational complexity.

In summary, our empirical analysis suggests that the wavelet-based model is a strong contender in the out-of-sample prediction and offers the second smallest hedging error in the hedging exercise during the sample period we study in this paper. This attests to the powerful approximation ability of the wavelet methodology. The superior forecasting performance of the wavelet-based model is due to the inherent de-noising and approximation ability of wavelets. 


\section{Conclusion}

This paper empirically evaluates the wavelet-based model and compares it with various option pricing models for their out-of-sample forecasting and hedging performance. The parametric SVJ model has the advantage that we can observe parameter estimates and assess the economic intuition of the risk factors they represent. Nonparametric methods including the neural network based and the wavelet-based models, on the other hand, possess greater flexibility in capturing the underlying asset price dynamics and the return distributions. The PBS model is very popular among practitioners due to its simplicity and flexibility in mitigating the volatility smile anomaly.

The data we use in this paper include daily end-of-day bid-ask midpoint of the DAX-30 index options. We show that in the out-of-sample forecasting exercise, the wavelet-based option pricing model consistently outperforms the SVJ. It also outperforms the PBS model for longterm options. The hedging performance of the wavelet method is the second best among all models considered apart from the NN. This suggests that the wavelet-based model is effective in revealing the risk-neutral MGF and hence useful for option pricing, forecasting and hedging. This empirical evaluation work therefore provides a solid basis for future research, such as utilizing the wavelet-based model for revealing risk preference and higher moments implied in option prices as well as gauging jump in the underlying asset with the wavelet method.

\section{Acknowledgement}

We thank the two reviewers for their detailed and helpful advice that greatly improved the paper. Any remaining errors and inaccuracies are ours.

\section{Appendix: Bilateral Laplace transform}

Following Haven et al. (2009), the bilateral Laplace transform is defined as follows. For a realvalued function $f(t)$ which is piecewise continuous on $[-\infty, \infty)$, its bilateral Laplace transformation is a 
complex valued function given by the following:

$$
\mathcal{L}\{f(t)\}(s)=F(s)=\int_{-\infty}^{\infty} f(t) e^{-s t} d t,
$$

where $s$ is a complex value and $\mathcal{L}$ denotes the Laplace transform operator. The inverse Laplace transform, denoted by $\mathcal{L}^{-1}\{F(s)\}(t)$, can be written as follows:

$$
\mathcal{L}^{-1}\{F(s)\}(t)=f(t)=\frac{1}{2 \pi i} \int_{c-i \infty}^{c+i \infty} F(s) e^{s t} d s,
$$

where $c$ is a specific real number.

Let $F(s)$ denote $\mathcal{L}\{f(x)\}(s)$ and $G(s)$ denote $\mathcal{L}\{g(x)\}(s)$, we have the properties of the Laplace transform summarized as follows:

1. Linearity

$$
\begin{gathered}
\mathcal{L}\{a f(x)+b g(x)\}(s)=a F(s)+b G(s) \\
\mathcal{L}^{-1}\{a F(s)+b G(s)\}(x)=a f(x)+b g(x) .
\end{gathered}
$$

2. Frequency shifting

$$
\begin{gathered}
\mathcal{L}\left\{e^{-l x} f(x)\right\}(s)=F(s+l), \forall l \in R ; \\
\mathcal{L}^{-1}\{F(s+l)\}(x)=e^{-l t} f(x), \forall l \in R .
\end{gathered}
$$

3. Time shifting

$$
\begin{gathered}
\mathcal{L}\left\{f\left(x-x_{0}\right)\right\}(s)=e^{-x_{0} s} F(s), \forall x_{0} \in R ; \\
\mathcal{L}^{-1}\left\{e^{-x_{0} s} F(s)\right\}(x)=f\left(x-x_{0}\right), \forall x_{0} \in R .
\end{gathered}
$$

4. Convolution

$$
\begin{gathered}
\mathcal{L}\{f(x) * g(x)\}=F(s) G(s) ; \\
\mathcal{L}^{-1}\{F(s) G(s)\}(x)=f(x) * g(x),
\end{gathered}
$$

where $*$ indicates the convolution operator on $f$ and $g$. This operator can be defined as (Bracewell (1999, page 25)),

$$
f * g \equiv \int_{-\infty}^{\infty} f(\tau) g(t-\tau) d \tau=\int_{-\infty}^{\infty} g(\tau) f(t-\tau) d \tau .
$$




\section{References}

Aït-Sahalia, Y., Duarte, J., 2003. Nonparametric option pricing under shape restrictions. Journal of Econometrics 116, 9-47.

Aït-Sahalia, Y., Lo, A., 1998. Nonparametric estimation of state-price densities implicit in financial asset prices. Journal of Finance 52, 490-547.

Andreou, P. C., Charalambous, C., Martzoukos, S. H., 2008. Pricing and trading European options by combining artificial neural networks and parametric models with implied parameters. European Journal of Operational Research 185, 1415-1433.

Andreou, P. C., Charalambous, C., Martzoukos, S. H., 2014. Assessing the performance of symmetric and asymmetric implied volatility functions. Review of Quantitative Finance and Accounting 42, 373-397.

Asgharian, H., 2011. A conditional asset-pricing model with the optimal orthogonal portfolio. Journal of Banking and Finance 35, 1027-1040.

Averkamp, R., Houdré, C., 2003. Wavelet thresholding for non-necessarily Gaussian noise: Idealism. Annals of Statistics, 110-151.

Bakshi, G., Cao, C., Chen, Z., 1997. Empirical performance of alternative option pricing models. Journal of Finance 52, 2003-2049.

Bakshi, G., Cao, C., Chen, Z., 2000. Pricing and hedging long-term options. Journal of Econometrics 94, $277-318$

Bakshi, G., Kapadia, N., 2003. Delta-hedged gains and the negative market volatility risk premium. Review of Financial Studies 16, 527-566.

Bali, T. G., Demirtas, K. O., 2008. Testing mean reversion in financial market volatility: Evidence from S\&P 500 index futures. Journal of Futures Markets 28, 1-33.

Bandi, C., Bertsimas, D., 2014. Robust option pricing. European Journal of Operational Research 239, $842-853$

Bates, D. S., 1996. Jumps and stochastic volatility: Exchange rate processes implicit in Deutsche mark options. Review of Financial Studies 9, 69-107.

Bates, D. S., 2000. Post-' 87 crash fears in the S\&P 500 futures option market. Journal of Econometrics $94,181-238$. 
Bayraktar, E., Poor, V., Sircar, R. H., 2004. Estimating the fractal dimension of the S\&P 500 index using wavelet analysis. International Journal of Theoretical and Applied Finance 5, 615-643.

Bengio, Y., 2009. Learning deep architectures for AI. Foundations and Trends in Machine Learning 2, 1-127.

Bennell, J., Sutcliffe, C., 2004. Black-Scholes versus artificial neural networks in pricing FTSE 100 options. Intelligent Systems in Accounting, Finance and Management 12, 243-260.

Berkowitz, J., 2002. Forecasting option prices with unobserved volatility and false models. Unpublished working paper.

Berkowitz, J., 2010. On justications for the ad hoc Black-Scholes method of option pricing. Studies in Nonlinear Dynamics and Econometrics 14, Article 4.

Birke, M., Pliz, K. F., 2009. Nonparametric option pricing with no-arbitrage constraints. Journal of Financial Econometrics 7, 53-76.

Black, F., Scholes, M., 1973. The pricing of options and corporate liabilities. Journal of Political Economy $81,637-659$.

Bliss, R. R., Panigirtzoglou, N., 2002. Testing the stability of implied probability density functions. Journal of Banking and Finance 26, 381-422.

Bracewell, R., 1999. The Fourier Transform and Its Applications. McGraw-Hill, US.

Cai, N., Kou, S., 2011. Option pricing under a mixed-exponential jump diffusion model. Management Science 57, $2067-2081$.

Capobianco, E., 1999. Statistical analysis of financial volatility by wavelet shrinkage. Methodology and Computing in Applied Probability 1, 423-443.

Capobianco, E., 2001. Wavelet transforms for the statistical analysis of returns generating stochastic processes. International Journal of Theoretical and Applied Finance 4, 511-534.

Christoffersen, P., Jacobs, K., 2004. The importance of the loss function in option valuation. Journal of Financial Economics 72, 291-318.

Christoffersen, P., S.L., H., Jacobs, K., 2009. The shape and term structure of the index option smirk: why multifactor stochastic volatility models work so well. Management Science 55, 1914-1932. 
Connor, J., Rossiter, R., 2005. Wavelet transforms and commodity prices. Studies in Nonlinear Dynamics and Econometrics 9, 1-22.

Coval, J. D., Shumway, T., 2001. Expected option returns. Journal of Finance 56, 983-1010.

Derman, E., Kani, I., 1994a. The volatility smile and its implied tree. Quantitative Strategies Research Notes Goldman Sachs, New York.

Derman, E., Kani, I., 1994b. Riding on the smile. Risk 7, 32-39.

Dong, M., He, D., 2007. Hidden semi-Markov models-based methodology for multi-sensor equipment health diagnosis and prognosis. European Journal of Operational Research 178, 858-878.

Dumas, B., Fleming, J., Whaley, R. E., 1998. Implied volatility functions: Empirical tests. Journal of Finance 53, 2059-2106.

Esteban-Bravo, M., Vidal-Sanz, J. M., 2007. Computing continuous-time growth models with boundary conditions via wavelets. Journal of Economic Dynamics and Control 31, 3614-3643.

Fernandez, V. P., 2006. The CAPM and value at risk at different time-scales. International Review of Financial Analysis 15, 203-219.

Gallegati, M., Gallegati, M., 2007. Wavelet variance analysis of output in G-7 countries. Studies in Nonlinear Dynamics and Econometrics 11, 1-23.

Garcia, R., Gencay, R., 2000. Pricing and hedging derivative securities with neural networks and a homogeneity hint. Journal of Econometrics 94, 93-115.

Garcia, R., Gençay, R., 2000. Pricing and hedging derivative securities with neural networks and a homogeneity hint. Journal of Econometrics 94, 93-115.

Gârleanu, N., Pedersen, L. H., Poteshman, A. M., 2009. Demand-based option pricing. Review of Financial Studies 22, 4259-4299.

Gençay, R., Selçuk, F., Whitcher, B., 2001a. Differentiating intra-day seasonalities through wavelet multi-scaling. Physica 289, 543-556.

Gençay, R., Selçuk, F., Whitcher, B., 2001b. Scaling properties of foreign exchange volatility. Physica $289,249-266$. 
Gençay, R., Selçuk, F., Whitcher, B., 2002. An introduction to wavelets and other filtering methods in finance and economics. Academic Press.

Gençay, R., Selçuk, F., Whitcher, B., 2003. Systematic risk and timescales. Quantitative Finance 3, $108-116$.

Gençay, R., Selçuk, F., Whitcher, B., 2005. Multiscale systematic risk. Journal of International Money and Finance 24, 55-70.

Genon-Catalot, V., Laredo, C.and Picard, D., 1992. Nonparametric estimation of the diffusion coefficient by wavelet methods. Scandinavian Journal of Statistics 19, 317-335.

Gradojevic, N., Genay, R., Kukolj, D., 2009. Option pricing with modular neural networks. IEEE Transactions on Neural Networks 20, 626-637.

Hagan, M., Menhaj, M., 1994. Training feedforward networks with the marquardt algorithm. IEEE Transactions on Neural Networks 5, 989-993.

Haven, E., Liu, X., Ma, C., Shen, L., 2009. Revealing the implied risk-neutral MGF from options: The wavelet method. Journal of Economic Dynamics and Control 33, 692-709.

Haven, E., Liu, X., Shen, L., 2012. De-noising option prices with the wavelet method. European Journal of Operational Research 222, 104112.

Heston, S. L., 1993. A closed-form solution for options with stochastic volatility with applications to bond and currency options. Review of Financial Studies 6, 327-343.

Hong, Y., Kao, C., 2004. Wavelet based testing for serial correlation of unknown form in panel models. Econometrica 72, 1519-1563.

Huang, J., Wu, L., 2004. Specification analysis of option pricing models based on time-changed Levy processes. Journal of Finance 59, 1405-1439.

Hutchinson, J. M., Lo, A., Poggio, T., 1994. A nonparametric approach to pricing and hedging derivative securities via learning networks. Journal of Finance 49, 851-889.

In, F., Kim, S., 2006. The hedge ratio and the empirical relationship between the stock and futures markets: A new approach using wavelet analysis. Journal of Business 79, 799-820.

Jensen, M., 1999. Using wavelets to obtain a consistent ordinary least squares estimator of the longmemory parameter. Journal of Forecasting 18, 17-32. 
Jensen, M., 2000. An alternative maximum likelihood estimator of long-memory processes using compactly supported wavelets. Journal of Economic Dynamics and Control 24, 361-387.

Kim, S., In, F., 2005. The relationship between stock returns and inflation: New evidence from wavelet analysis. Journal of Empirical Finance 12, 435-444.

Ko, K., Vannucci, M., 2006. Bayesian wavelet analysis of autoregressive fractionally integrated movingaverage processes. Journal of Statistical Planning and Inference 10, 3415-3434.

Lada, E. K., Wilson, J. R., 2006. A wavelet-based spectral procedure for steady-state simulation analysis. European Journal of Operational Research 174, 1769-1801.

Lajbcygier, P., Boek, C., Palaniswami, M., 1996. Comparing conventional and artificial neural network models for the pricing of options on futures. Neurovest Journal 4, 16-24.

Lien, D., Shrestha, K., 2007. An empirical analysis of the relationship between hedge ratio and hedging horizon using wavelet analysis. Journal of Futures Markets 27, 127-150.

Ma, C., 2011. Advanced Asset Pricing Theory. Imperial College Press.

Mallat, S., 1999. A Wavelet Tour of Signal Process. Academic Press.

Manchaldore, J., Palit, I., Soloviev, O., 2010. Wavelet decomposition for intra-day volume dynamics. Quantitative Finance 10, 917-930.

Matache, A.-M., Nitsche, P.-A., Schwab, C., 2005. Wavelet Galerkin pricing of American options on Levy driven assets. Quantitative Finance 5, 403-424.

Mitra, S., 2006. A wavelet filtering based analysis of macroeconomic indicators: The Indian evidence. Applied Mathematics and Computation 175, 1055-1079.

Nikkinen, J., pynnönen, S., Ranta, M., Vähämaa, S., 2011. Cross-dynamics of exchange rate expectations: A wavelet analysis. International Journal of Finance \& Economics 16, 205-217.

Ortiz-Gracia, L., Oosterlee, C. W., 2016. A highly efficient Shannon wavelet inverse Fourier technique for pricing European options. SIAM Journal on Scientific Computing 38, B118-B143.

Pan, J., 2002. The jump-risk premia implicit in options: Evidence from an integrated time-series study. Journal of Financial Economics 63, 3-50. 
Percival, D. B., Walden, A. T., 2000. Wavelet methods for time series analysis. Cambridge University Press.

Ramsey, J. B., Lampart, C., 1998a. The decomposition of economic relationship by time scale using wavelets: Expenditure and income. Studies in Nonlinear Dynamics and Econometrics 3, 23-42.

Ramsey, J. B., Lampart, C., 1998b. Decomposition of economic relationship by time scale using wavelets: Money and income. Macroeconomic Dynamics 3, 23-42.

Ren, S., He, K., Girshick, R., 2016. Faster R-CNN: Towards real-time object detection with region proposal networks. IEEE Transactions on Pattern Analysis and Machine Intelligence 39, 1137 - 1149.

Rubinstein, M., 1994. Implied binomial trees. Journal of Finance 49, 771-818.

Santa-Clara, P., Yan, S., 2010. Crashes, volatility, and the equity premium: Lesson from the S\&P 500 options. Review of Economics and Statistics 92, 435-451.

Shimko, D., 1994. Bounds of probability. Risk 6, 33-37.

Sun, E. W., Meinl, T., 2012. A new wavelet-based denoising algorithm for high-frequency financial data mining. European Journal of Operational Research 217, 589-599.

Weron, R., 2009. Heavy tails and regime-switching in electricity prices. Mathematical Methods of Operations Research 69, 457-473.

Wong, H., Lo, Y., 2009. Option pricing with mean reversion and stochastic volatility. European Journal of Operational Research 197, 179-187.

Yao, J., Li, Y.and Tan, C., 2000. Option price forecasting using neural networks. International Journal of Management Science 28, 455-466.

Zapart, C., 2002. Stochastic volatility options pricing with wavelets and artificial neural networks. Quantitative Finance 2, 487-495. 
Table 1. Summary statistics of options data

This table provides summary statistics of options written on the German DAX-30 index. Moneyness is defined as X/S. The sample period is from January 2, 2009, to December 28, 2012.

\begin{tabular}{|c|c|c|}
\hline & Call options & Put options \\
\hline Total No. of options & 65867 & 95895 \\
\hline Total No. of trading days & 1004 & 1004 \\
\hline \multicolumn{3}{|c|}{ Panel A. Short-term options } \\
\hline Avg No. per day & 36.59 & 54.05 \\
\hline Range of moneyness & {$[1,1.9144]$} & {$[0.2945,1]$} \\
\hline Moneyness $<0.94$ & & $37376(68.87 \%)$ \\
\hline $0.94<=$ Moneyness $<0.97$ & & $8406(15.49 \%)$ \\
\hline Moneyness $>=0.97$ & & $8487(15.64 \%)$ \\
\hline Moneyness $<1.03$ & $8606(23.43 \%)$ & \\
\hline $1.03<=$ Moneyness $<1.06$ & $8581(23.36 \%)$ & \\
\hline Moneyness $>=1.06$ & $19548(53.21 \%)$ & \\
\hline \multicolumn{3}{|c|}{ Panel B. Medium-term options } \\
\hline Avg No. per day & 16.26 & 22.47 \\
\hline Range of moneyness & {$[1,1.6033]$} & {$[0.2359,1]$} \\
\hline Moneyness $<0.94$ & & $17071(76.51 \%)$ \\
\hline $0.94<=$ Moneyness $<0.97$ & & $2594(11.63 \%)$ \\
\hline Moneyness $>=0.97$ & & $2648(11.87 \%)$ \\
\hline Moneyness < 1.03 & $2648(16.39 \%)$ & \\
\hline $1.03<=$ Moneyness $<1.06$ & $2648(16.39 \%)$ & \\
\hline Moneyness $>=1.06$ & $10864(67.23 \%)$ & \\
\hline \multicolumn{3}{|c|}{ Panel C. Long-term options } \\
\hline Avg No. per day & 19.12 & 23.64 \\
\hline Range of moneyness & {$[1,1.9144]$} & {$[0.1388,1]$} \\
\hline Moneyness $<0.94$ & & $18711(78.81 \%)$ \\
\hline $0.94<=$ Moneyness $<0.97$ & & $2379(10.02 \%)$ \\
\hline Moneyness $>=0.97$ & & $2653(11.17 \%)$ \\
\hline Moneyness $<1.03$ & $2633(13.74 \%)$ & \\
\hline $1.03<=$ Moneyness $<1.06$ & $2517(13.14 \%)$ & \\
\hline Moneyness $>=1.06$ & $14008(73.12 \%)$ & \\
\hline
\end{tabular}




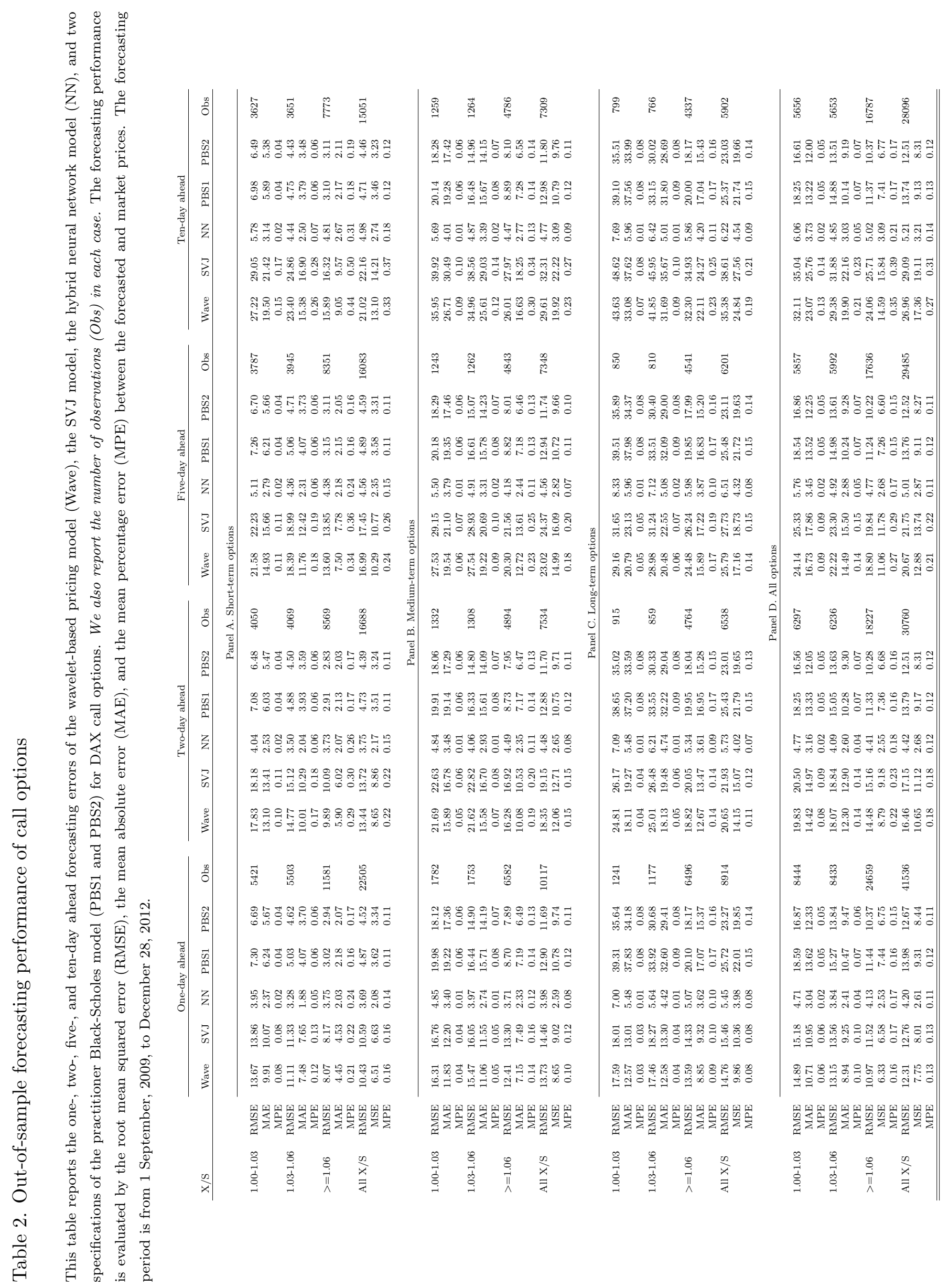




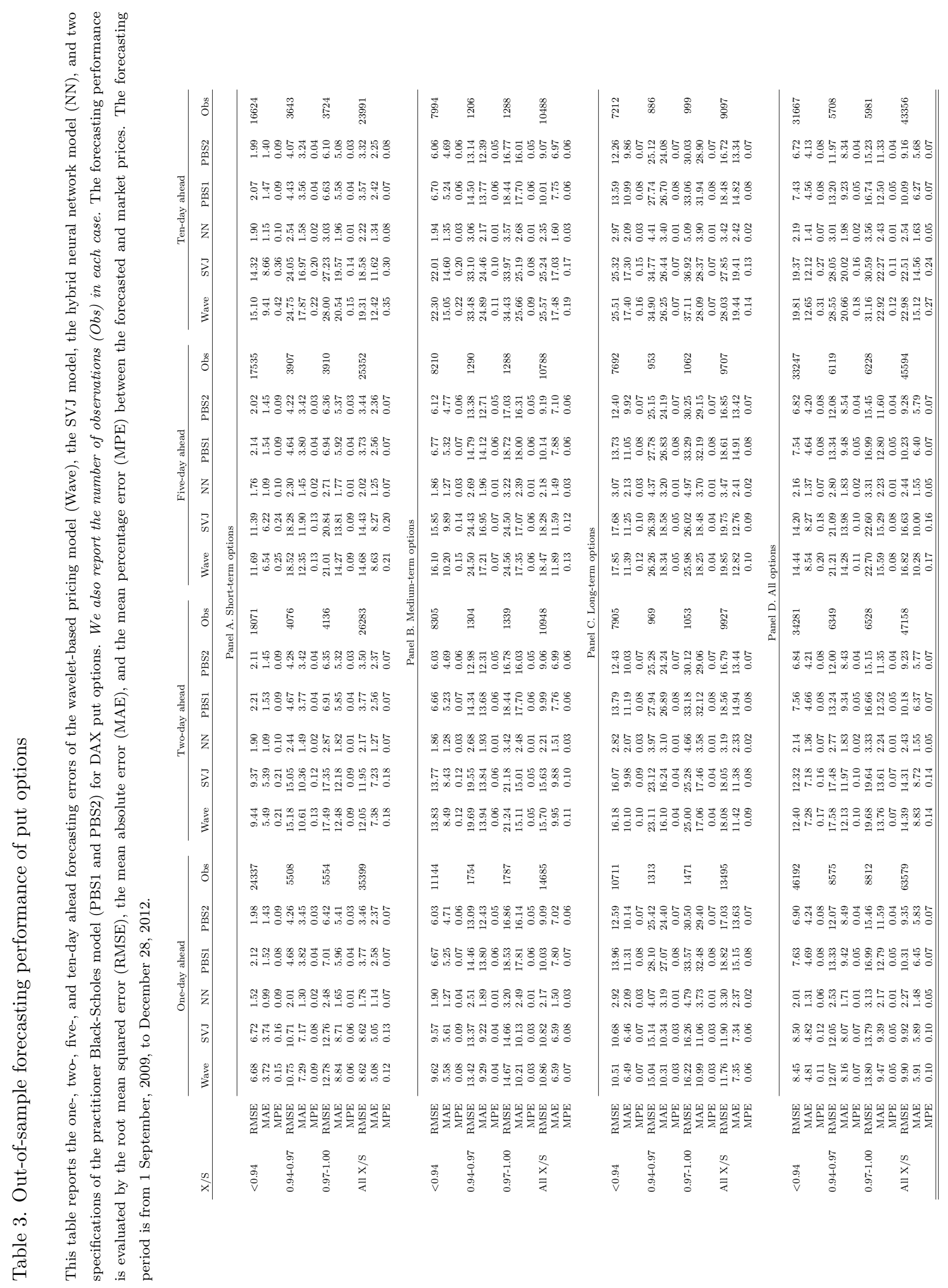




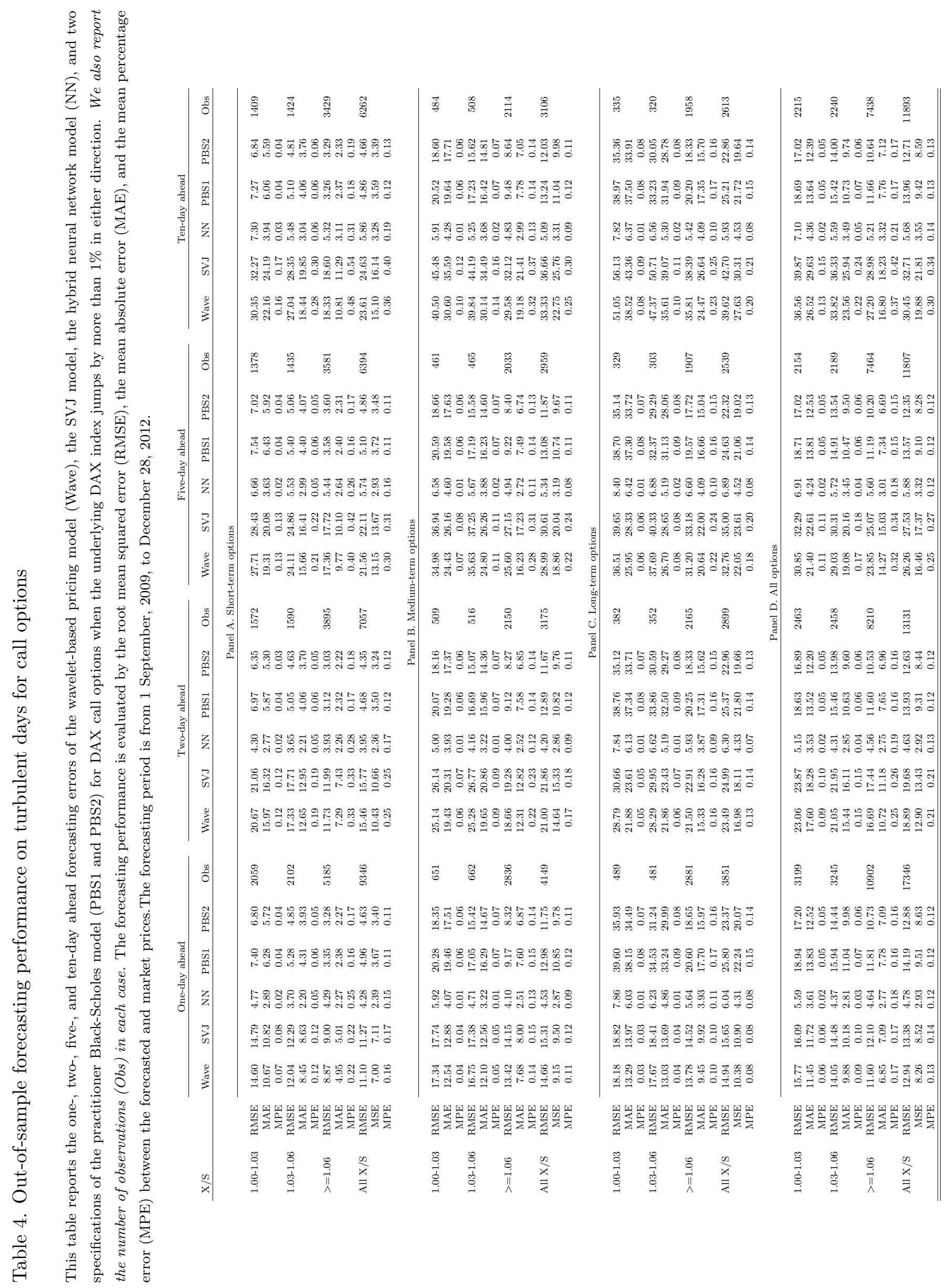




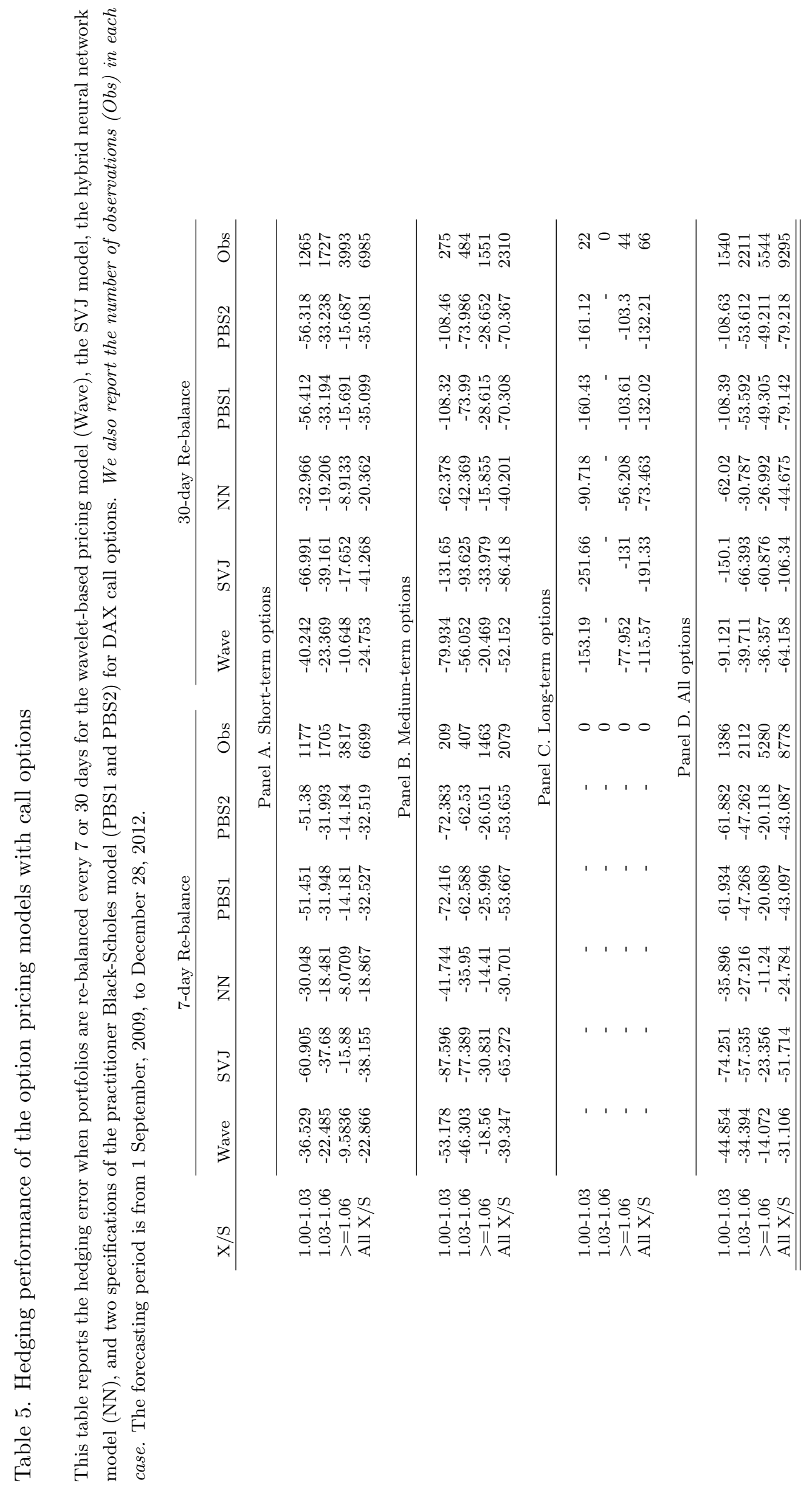

